\title{
Developmental Origins of Health Span and Life Span: A Mini-Review
}

\author{
Joshua D. Preston ${ }^{a}$ Leryn J. Reynolds ${ }^{b}$ Kevin J. Pearson ${ }^{a}$ \\ ${ }^{a}$ Pharmacology and Nutritional Sciences, University of Kentucky College of Medicine, Lexington, $K Y$; ${ }^{b}$ Department \\ of Human Movement Sciences, Old Dominion University Darden College of Education, Norfolk, VA, USA
}

\section{Keywords}

Aging · Developmental origins of health and disease · Developmental programming · Disease - Epigenetics . Exercise $\cdot$ Longevity $\cdot$ Pregnancy

\begin{abstract}
Background: A vast body of research has demonstrated that disease susceptibility and offspring health can be influenced by perinatal factors, which include both paternal and maternal behavior and environment. Offspring disease risk has the potential to affect the health span and life span of offspring. Key Findings: Various maternal factors, such as environmental toxicant exposure, diet, stress, exercise, age at conception, and longevity have the potential to influence age-associated diseases such as cardiovascular disease, obesity, diabetes, and cancer risk in offspring. Paternal factors such as diet, age at conception, and longevity can potentially impact offspring health span and life span-reducing traits as well. Practical Implications: Continued research could go a long way toward defining mechanisms of the developmental origins of life span and health span, and eventually establishing regimens to avoid negative developmental influences and to encourage positive interventions to potentially increase life span and improve health span in offspring.
\end{abstract}

(c) 2018 S. Karger AG, Basel

\section{KARGER}

๑ 2018 S. Karger AG, Basel

E-Mail karger@karger.com

www.karger.com/ger

\section{Introduction}

The field studying the developmental origins of health and disease hypothesis has been an emerging and rapidly growing area of research for several decades. This hypothesis strongly suggests that multiple factors during prepregnancy, gestation, and the early postnatal period influence long-lasting disease susceptibility in offspring [1]. Markers of offspring health and even life span can be influenced by numerous maternal and paternal factors, and it is likely that many of these parental effects are transferred by multiple molecular mechanisms, including epigenetic regulation [1].

The primary purpose of this mini-review is to highlight areas of the existing literature where the maternal and early postnatal environments influence later life obesity, type 2 diabetes mellitus (T2DM), and cardiovascular disease (CVD) risk in offspring because these diseases have been shown to substantially reduce life expectancy $[2,3]$. However, we will also briefly explore additional disease outcomes and less studied areas such as paternal influences prior to conception. While most work has focused on negative effects on offspring disease, there is a growing body of evidence asserting that positive interventions, such as maternal exercise, could be used to improve offspring health span [4]. Regardless, preconcep- 
tion, pregnancy, and early postnatal life appear to be a short-term window where healthy behaviors could provide a lifetime of health benefits to offspring.

\section{Parental Diet, Obesity, and Diabetes}

Hales and Barker hypothesized in a 1992 paper that maternal undernutrition could retard the growth of fetal beta cells in utero and subsequently lead to T2DM and metabolic syndrome in adult life; this hypothesis is known as the "thrifty phenotype hypothesis," because a fetus can be forced to assume its most thrifty phenotype tailored to a maternally undernourished environment [5]. Barker et al. [6] provided a subsequent review on how in utero nutrition affected offspring CVD risk and development. These early reviews sparked intense research into how the maternal milieu could influence offspring phenotypes, and this field later fell under the developmental origins of health and disease hypothesis [1]. Multiple retrospective analyses have been performed in humans, and subsequent animal studies have attempted to delineate mechanisms. For an excellent review on work being performed in the developmental origins of health and disease field, see Wadhwa et al. [1], and for an overview of epigenetics and further reading on epigenetic mechanisms, see Holliday [7].

\section{Human Studies}

Maternal Diabetes

A vast body of literature has reflected that diabetes during pregnancy can have long-lasting metabolic effects on offspring. One prime example of this can be seen in studies conducted in Pima Indians. These studies indicate that maternal diabetes poses a significant risk to offspring development of T2DM, higher systolic blood pressure and hemoglobin A1c, and greater rates of obesity [8-10]. Drawing on a large body of evidence, Simeoni and Barker [11] assert in their review that exposure to high intrauterine glucose concentration can independently influence T2DM risk in offspring, irrespective of whether or not a mother has diabetes. Thus, increased intrauterine glucose exposure may contribute to the developmental origins of later disease [11]. For a meticulous review on both human and animal studies being performed on maternal diabetes, mechanisms, and possible epigenetic modes of transmission, see Ma et al. [12].

Gestational Weight Gain and Maternal Obesity

Obese individuals represent a rapidly growing demographic in the global population, and this disease has the potential to affect offspring disease risk [13]. For additional information on maternal obesity and its impact on offspring health and disease, see Lustig's thorough review [13]. In regards to maternal body mass index (BMI), Olson et al. [14] found that mothers with overweight or obese BMIs during early pregnancy had children that were significantly more likely to be overweight at 3 years of age. Not only was early-pregnancy BMI correlated with offspring overweight at 3 years of age, but excessive weight gain (more than the Institute of Medicine's recommendation per BMI category) led to a higher risk of overweight offspring at 3 years of age [14]. Excessive weight gain during pregnancy by mothers that were either overweight or obese in early pregnancy aggravated this effect in offspring [14]. These findings suggest that obesity prior to pregnancy serves as a contributor to offspring obesity, especially if excessive weight gain occurs during pregnancy. The pathophysiology behind the modulation of offspring health by gestational weight gain and maternal obesity remains somewhat unknown; however, for an outstanding review on human and animal models which explore mechanisms, see Poston [15].

\section{Maternal Undernutrition}

The Dutch famine was a period of intense caloric deprivation in a specifically defined period of time during the winter of 1944-1945. Caloric intake often dipped below 800 calories per day per person, and pregnant women were not excluded. As unfortunate as this event was, it served as an instance to examine the specific effects of defined maternal caloric deprivation on offspring health in humans. One significant finding from examining the offspring was famine-exposed babies during certain periods of gestation had lower birth weight [16]. Low birth weight has been shown to be associated with CVD and metabolic syndrome later in life $[6,16,17]$.

Interestingly, the offspring health effects of maternal caloric deprivation during the Dutch famine appear to be time specific as to when the restriction occurred during pregnancy [16]. For example, Painter et al. [16] found that exposure to famine during late pregnancy was most closely associated with impaired glucose tolerance in offspring (measured by significantly higher 120-minute glucose and insulin levels). Exposure to famine during mid gestation correlated with increased incidence of microalbuminuria and obstructive airway disease in offspring [16]. Lastly, babies exposed to famine during early gestation, while not having lower than average birth weight compared to their mid-late gestation exposed counterparts, displayed increased coronary heart disease and 
obesity, elevated lipids, and altered clotting later in life [16]. In cases where negative organ system outcomes can be seen in offspring as a result of time-specific exposure, Painter et al. [16] hypothesized that energy deprivation retards growth of the specific system that is affected. For example, since the second trimester is a period of rapid nephron and bronchial development, undernutrition during these periods could lead to negative, organ-specific health outcomes in adult offspring such as microalbuminuria or obstructive airway disease [16].

Barker et al. [6] suggest that low birth weight in offspring of mothers who are calorically restricted in late pregnancy could be due to placental function taking precedence over fetal growth, and such low birth weight may predispose offspring to CVD. However, despite low birth weight being associated with CVD and metabolic syndrome $[6,17]$, many of the findings regarding health outcomes of adult offspring from Painter et al. [16] were independent of the birth weight, which suggests that caloric deprivation may play an independent role in the fetal origins of adult diseases. Epigenetic regulation, and particularly DNA methylation, seems to potentially influence this process, as Heijmans et al. [18] found that offspring exposed to the Dutch famine 6 decades prior displayed persistent DNA hypomethylation of the differentially methylated region of the insulin-like growth factor 2 gene in comparison to same-sex, unexposed siblings.

It is important to note that while low birth weight has a correlation with later life metabolic dysfunctions, it may not be a causal mechanism. Rather, it is likely a cofactor that is accompanied by the modification of other mechanisms that set the trajectory for later life metabolic function, as Barker et al. [6] assert that birth weight is simply a surrogate for programmed phenotypes. For example, findings from the 1958 British Birth Cohort indicate that prenatal exposures certainly have the power to influence later-life glucose metabolism; however, this was mediated largely through adiposity in offspring rather than birth weight [19]. It is possible that low birth weight babies experience a phenomenon known as "catch-up growth," which may be predisposing them to increased CVD risk and metabolic disease later in life (mouse models which test this directly are discussed in the section Catch-Up Growth).

Unfortunately, fetal nutritional restriction does not just increase offspring CVD and metabolic disease rates, but can impact cancer risk and cognitive function later in life as well. Painter et al. [20] found a significantly higher incidence of breast cancer in women who were born to mothers exposed to Dutch famine during early pregnancy. Additionally, adult offspring of mothers exposed to the Dutch famine during early pregnancy exhibit lower performance on a "selective attention task" at age 56-59 [21]. This may suggest an increased rate of age-associated deterioration and cognitive decline in offspring exposed during early gestation [21]. Mechanisms that govern these two phenomena remain speculative. Taken together, these findings are indicative that prenatal undernutrition does not limit its effects to metabolic damage in offspring, but can also affect other age-associated diseases which could impact health span and life span.

\section{Paternal and Grandpaternal Diet}

Paternal diet appears to have the potential to alter both positive and negative offspring phenotypes. Studies of Swedish birth cohorts found that fathers who experienced low food availability during their "slow growth period" (defined as ages 9-12 for men) had offspring that showed lower CVD mortality [22]. Moreover, subjects in one Swedish birth cohort displayed increased survival if their paternal grandfather experienced low food availability during their slow growth period. However, negative phenotypes can arise as well, as excess food availability had the opposite effect [23]. Additionally, subjects displayed increased diabetes mortality when the paternal grandfather lived through high food availability during their slow growth period [22]. The mechanisms behind the effect of grandpaternal diet remain elusive, but both Kaati et al. [22] and Bygren et al. [23] have suggested genomic imprinting as a likely explanation. For an excellent discussion on genomic imprinting and potential transgenerational effects, see Pembrey [24]. Food restriction can have a very different effect depending on the timing, as Veenendaal et al. [25] found that fathers who were undernourished in gestation (F1) due to the Dutch famine had offspring (F2) that were more obese. Interestingly, this effect was not seen in offspring of mothers undernourished in utero. Taken together, these findings indicate that paternal and grandpaternal diet may lead to both positive and negative phenotypes in offspring, although this effect depends largely on timing and feeding behavior. For rat models that test the effect of high-fat diet on transgenerational metabolism, see Chambers et al. [26].

Model Systems to Study Developmental Origins in Humans

Our laboratory has recently begun using neonatal skin to study in utero exposures and possible mechanisms that could contribute to later disease risk [27]. Primary dermal 
fibroblasts from foreskin samples can be used as a living, functional system to study the potential insulin signaling, adipogenesis, or stress response differences resulting from different in utero exposures [27]. Further, skin biopsies could be taken in the same subject later in life for longitudinal measures as the person ages.

\section{Animal Studies}

Animal studies provide for more specific interventions compared to human studies, which are frequently limited to retrospective data analyses. The following is not allinclusive but attempts to highlight relevant animal studies in regards to perinatal influences on offspring age-associated diseases, health span, and life span.

\section{Maternal Dietary Interventions}

Samuelsson et al. [28] found that female mice fed a high-fat diet prior to mating and during pregnancy and nursing had offspring that exhibited increased adiposity, glucose intolerance, and hypertension once weaned onto regular diets. In addition, Alfaradhi et al. [29] found high-fat diet-fed female mice had offspring that exhibited non-alcoholic fatty liver disease, oxidative damage in the liver of these offspring, and insulin resistance at 8 weeks of age, prior to the development of increased adiposity; thus, Alfaradhi et al. [29] propose that oxidative damage could be a mechanism by which the offspring develop non-alcoholic fatty liver disease and insulin resistance. Additionally, Taheripour et al. [30] demonstrated in a swine model that offspring of mothers who consumed high-energy diets during pregnancy displayed impaired vascular function. This was expressed in the form of reduced vasorelaxation in offspring femoral arteries (measured by endothelial-dependent and -independent vasorelaxation), which may be a risk factor for atherosclerosis. Indeed, similar mechanisms may be at play in the impaired vascular function of 3-month-old mouse offspring of high-fat diet-fed dams, as these mice were hypertensive and exhibited endothelial dysfunction [28]. These studies indicate that maternal high-fat and/ or high-energy diets can lead to negative and potentially life span-reducing phenotypes in offspring. Conversely, positive maternal dietary interventions have been demonstrated in mouse models in the past. For example, Harman and Eddy's study found that dams that were fed a diet supplemented with the antioxidant 2-mercaptoethylamine during pregnancy had offspring who experienced significantly increased average life spans compared to controls (14.7 and $8.6 \%$ for male and female offspring, respectively) [31].
Early Body Size, Crowded-Litter, and Preweaning Protein Restriction

While many dietary interventions in the developmental origins of health and disease field depend on maternal diet during pregnancy, a significant body of literature asserts that perinatal influences such as postnatal caloric restriction and maternal low-protein diets (during lactation) can have significant effects on offspring life span. It has been shown that mice that are calorically restricted in early life (first 20 days) via 50\% litter enlargement show an $18 \%$ increase in median life span compared to controls [32]. Ozanne and Hales [33] demonstrated that male mice that were nursed by a mother that was on a lowprotein diet during lactation outlived controls (814 vs. 765 days of average age at death).

In a laboratory setting, caloric restriction enacted during adulthood has been shown to delay the onset of ageassociated diseases and improve survival in numerous species. The emerging literature discussed above suggests that a similar effect of life span extension can be seen from caloric restriction and protein restriction in early postnatal life, but not in utero, even if mice are weaned onto a normal, ad libitum diet after weaning [32, 33].

Male mice nursing from low-protein-fed dams challenged with an obesity-inducing diet after weaning showed significantly increased life span compared to controls fed the same obesity-inducing diet (807 vs. 715 days of average age at death) [33]. Also, adult rats born to dams that were protein restricted during lactation showed lower body weight and adiposity as well as improved glucose homeostasis compared to controls [34]. Interestingly, offspring nursed by low-protein-fed dams or born into large litters (both early mechanisms of calorie restriction) are seemingly not programmed to restrict their calorie intake throughout later life, as adult postnatal low-protein rat offspring showed no differences in food intake compared to controls [34], and crowded-litter male mice also showed no differences in food intake compared to control mice at 6 months of age [35]. This may suggest that the positive effects seen in enlarged litter and postnatal low-protein offspring are not due to lower food intake, but rather early metabolic programming.

It is possible that the mechanisms that mediate perinatal metabolic programming fall under the general phenomenon of body size-dependent life span trajectory. Indeed, postnatal low-protein rats showed persistently lower body weights from nursing to adulthood [34]. It has been established that decreased body size (ages 2-24 months) can predict life span in mice [36], but this should 
not be confused with low birth weight. For further reading on animal models of early nutritional restriction and aging, see Davis et al. [37].

\section{Catch-Up Growth}

Mouse models have demonstrated that male mice which are undernourished prenatally via maternal lowprotein diet and then cross-fostered to control-fed dams experience a marked decrease in average age at death compared to controls. Cross-fostering to control fed dams results in greater nutritional intake directly after prenatal undernutrition, and initiates what Ozanne and Hales have deemed "catch-up growth," (mentioned above as being potentially at play in humans with low birth weights) [33]. Furthermore, this difference was exacerbated if mice were placed on obesogenic diets, as mice exposed to prenatal caloric restriction still had significantly shorter life spans on average than mice that were not exposed and put on the same obesogenic diet [33]. A version of catch-up growth may partially be at play in humans and the development of later life metabolic syndrome and other comorbid diseases such as CVD or obesity, as Eriksson et al. [38] found those who were thin at birth but experienced catch-up growth had increased CVD morbidity as adults.

Sirtuin 1 (SIRT1) is a protein implicated to be involved in some of the life span-extending effects of calorie restriction. While numerous pathways and mechanisms are likely involved, there are data to suggest that decreased SIRT1 protein expression may play a role in the decreased life span that arises from catch-up growth, as Chen et al. [39] found that male mice that experienced catch-up growth displayed decreased SIRT1 protein expression in muscle tissue compared to controls.

\section{Environmental Toxicants}

Prenatal exposures to multiple environmental toxicants pose significant risk to the health of offspring in both mouse models and humans. Correlations have been made between prenatal cigarette smoke exposure and later disease in humans $[40,41]$ and prenatal cigarette smoke and environmental toxicant exposure and life span-decreasing diseases in rodents $[42,43]$.

\section{Cigarette Smoke}

Maternal cigarette smoke exposure remains one of the most persistent and ubiquitous prenatal hazards. In 2014, $8.4 \%$ of US women smoked during pregnancy despite the well-explored negative health effects on offspring [44]. In humans, maternal smoking has been shown to play a role in the offspring development of diseases that reduce life expectancy, such as obesity. For example, a systematic review and meta-analysis found a positive association of maternal smoking with childhood overweight, as well as childhood obesity [41]. It seems that this risk also carries into adulthood, as one study found an odds ratio for obesity of 1.56 in men and 1.41 in women at age 33 whose mothers smoked during pregnancy; these differences remained after adjustment for confounders [40]. Additionally, babies from the same cohort were found to have lower birth weight if their mother smoked during pregnancy [40]. Maternal smoking's effect on offspring metabolism has also been tested in mouse models. Chen et al. [43] found that in dams exposed to high-fat diet/chow and sham/cigarette during pregnancy, offspring of dams exposed to smoke were more glucose intolerant compared to controls, and this effect was exacerbated by maternal high-fat diet.

\section{Polychlorinated Biphenyls}

Polychlorinated biphenyls (PCBs) are a prevalent and common environmental contaminant, contained, for example, in insulating material, plastic softeners, and electrical equipment. Even though PCBs were banned in the 1970s, exposure still continues today due to the lasting ability of PCBs to contaminate groundwater and our food supply. PCBs are considered endocrine disrupting chemicals; thus, PCBs may contribute to the development of obesity and metabolic disease [45]. We have previously shown that perinatal PCB126 exposure can have sex-specific effects on offspring body composition. Female offspring that were exposed perinatally to PCBs had a dose-dependent decrease in percent lean tissue and increase in adiposity, while males had a significant reduction in lean mass in response to perinatal environmental contaminant exposure [42]. Current studies in our laboratory are exploring the long-term effects of in utero and postnatal exposure to PCBs on adiposity and glucose homeostasis as offspring age. Since obesity and impaired glucose homeostasis can decrease life expectancy, PCBs and other environmental exposures (such as 4-nonylphenol and bisphenol A) could potentially be a perinatal influence of life span and health span.

\section{Maternal Social Stress and Mental Health}

There is an emerging field of literature that explores the effects of maternal social stress and mental health during pregnancy on offspring health and disease. Plant et al. 
[46] found that prenatal depression was associated with greater rates of clinically significant inflammation in adult offspring as measured by circulating high-sensitivity C-reactive protein independent of adult depression or childhood maltreatment. Also, trauma and extreme stress experienced by a mother during gestation may be a predictor of insulin resistance and higher BMI in adult offspring, as Entringer et al. [47] showed both of these traits to be significantly elevated in offspring born to mothers who experienced such stress. Additionally, King et al. [48] found that some maternal social disadvantages increased methylation at the differentially methylated regions of the maternally expressed gene 3 (MEG3) in offspring. Because MEG3 expression has been shown to have an inverse relationship with incidence of several cancer types, methylation of the MEG3 differentially methylated regions could potentially predispose offspring to cancer [48]. Together, these initial data could lead to speculation that maternal adversity, stress, or social disadvantage could decrease offspring life span due to cancer risk, inflammation, or metabolic disease. For an excellent review of animal and human work being done on maternal stress and developmental origins, as well as potential mechanisms, see Brunton [49].

\section{Parental Longevity and Age at Conception, Telomere Length, and Season of Birth}

While the prior sections have focused on factors that involve the in utero and postnatal period, this section will outline studies that examine influences on offspring life span and longevity such as season of birth, maternal and paternal age at conception, telomere length, and maternal and paternal longevity.

\section{Season of Birth}

Season of birth has been shown to have an influence on longevity. For example, in a study of 1880-1895 born US centenarians, it was demonstrated that the odds of becoming a centenarian increased significantly if the month of birth was in the second half of the year (especially September-November) as opposed to the first half of the year (specifically March) [50]. Various prenatal and postnatal environmental factors can fluctuate as a function of season of birth, as Gavrilov and Gavrilova [50] propose that improved maternal nutrition, lower rates of seasonal infection for newborns, mild ambient temperature in early life, and potential seasonal differences in sperm quality at conception might account for the in- crease in likelihood of fall babies becoming centenarians. Some evidence has shown that sperm and semen quality can be affected by season and age [51]. Future research should address questions of whether sperm or semen quality could influence the health and life span of offspring. Contrarily, adverse perinatal environment could potentially act as an early biological insult to a developing fetus or baby. These data specifically corroborate the "high initial damage load hypothesis," which posits that events that cause early damage in an organism can set its life span trajectory [52]. These effects may be lessened due to improvements in nutrition and disease prevention in the US; nevertheless, these findings still provide some proof of concept for the developmental origins of life span.

\section{Paternal and Maternal Longevity and Age at Conception}

Moreover, paternal and maternal longevity $(80+$ years of age) was associated with marked increases in the likelihood for offspring to become a centenarian [53]. Thus, this study suggests that parental longevity can be a heritable and independent predictor of offspring longevity. Additionally, parental age at birth/conception can be associated with offspring longevity. Gavrilov and Gavrilova [53] reviewed multiple studies that have found a positive effect of younger maternal age at birth increasing the chances of offspring becoming a centenarian and also paternal age at conception being inversely proportional with longevity in female offspring [54]. It is important to note that since the time at which parents have their first child is increasingly delayed until later ages in the Western world [55], paternal and maternal age at conception could still serve as a significant factor influencing offspring life span. Additionally, a study in mice supported the notion of younger maternal age at birth prolonging life span in female offspring [56]. Interestingly, younger maternal age did not have the same effect in male offspring, and older maternal age at birth even prolonged life span of male offspring in some strains [56].

\section{Telomere Length}

Telomeres are structures of DNA and protein located at the end of the chromosome that help to stabilize and protect the genome. Telomere length has been implicated to play a role in aging, as telomere length decreases with age, and shortened telomere length can affect life span and age-related disease susceptibility. A number of studies have been performed to examine the paternal and maternal influences on telomere length. High maternal pre- 
pregnancy BMI independently led to shorter telomere length in newborn offspring (measured in placenta and cord blood) [57]. A study in a homogenous Amish population found that paternal telomere length was associated with telomere length in offspring, and paternal life span was correlated with telomere length in female offspring [58]. These data indicate a degree of prenatal and parental influence on offspring telomere length, and emphasize the importance for further research to explore such influences on telomere length and their effect on life span and health span.

\section{Exercise}

While a majority of this review has focused attention on negative parental behaviors and factors, this section will briefly highlight perinatal exercise as a positive intervention. For more detailed reading on the impact of maternal exercise on offspring health, refer to the review by Blaize et al. [4].

Our laboratory has found that in mouse and rat models, maternal exercise (voluntary wheel running vs. sedentary) significantly improves both insulin sensitivity and glucose homeostasis in offspring $[59,60]$. Further, we found that male mice born to mothers that exercised during pregnancy showed lower fat mass and greater lean mass compared to offspring of sedentary pregnancies [59]. Additionally, maternal exercise during pregnancy conferred a protective effect in male rat offspring against non-alcoholic fatty liver disease in response to high-fat diet [61]. We found that exercise during pregnancy can help stave off the effects of the high-fat diet on the pregnant dam herself [62]. Our findings from this study showed that dams fed a high-fat diet that were exercised showed comparable weight, glucose disposal, and body fat mass with control sedentary mice fed a standard diet [62]. Since diabetic and obese pregnancies can lead to negative traits in offspring (see the sections Maternal Diabetes and Gestational Weight Gain and Maternal Obesity), this study suggests exercise during pregnancy may be an especially effective intervention in obese pregnant women, although this needs to be tested in humans.

Other work has found a strong association between maternal exercise and improved cardiovascular function in human and porcine offspring $[63,64]$. In humans, it was found that maternal exercise was associated with increased fetal heart rate variability and decreased heart rate, indicative of improved cardiac autonomic control [64]. In porcine models, maternal exercise resulted in im-

Perinatal Influences on Disease

Development proved thoracic aorta endothelium-dependent vasorelaxation in female offspring [63]. Findings on maternal exercise in animal models are not solely limited to metabolic or vascular programming; improvements in cancer risk and Alzheimer's risk in offspring have been observed as well $[65,66]$.

These initial data in animal models indicate that prenatal exercise can lower the risk for age-related diseases which could increase life expectancy, and further work in animals and humans could delineate the specific mechanisms at work and underscore the importance of maternal exercise as a positive intervention in offspring to increase health span and even life span.

\section{Conclusions}

The growth of the developmental origins of health and disease field has opened new avenues for research and has led the scientific world to rethink traditional models of vertical inheritance. It is evident from the above literature that a variety of perinatal influences can have long-lasting effects on traits which could affect health span and potentially increase or reduce life expectancy in offspring. While we focused mainly on metabolic disease and CVD risk, many studies on cancer and neurodegenerative disease can be found in the literature.

This review asserts the importance of gestational health worldwide. With diabetic and obese pregnancies common in the developed world and fetal malnutrition still common in developing countries, it is extremely important for special emphasis to be placed on maternal health. The vast and growing body of literature that examines the extreme sensitivity of the maternal milieu and the perinatal period necessitates greater research effort and funding dedicated towards the developmental origins of health and disease hypothesis and epigenetics. Moreover, an increase in large birth cohort studies involving (epi)genomic testing would allow for an even greater body of knowledge in this field. Conclusive results that elucidate the mechanisms at work in various developmental origins phenomena could provide groundbreaking innovation in optimizing pregnancy feeding and exercise regimens, creating pharmacogenomic interventions, and pinpointing both paternal and maternal lifestyle adjustments that could have significant longterm effects on health span and life span in offspring. Studies reviewed in this article have shown promising temporary maternal interventions that help improve health in offspring. The treatment of maternal diabetes 
and gestational diabetes mellitus, maternal smoking cessation, exercise interventions in obese women who plan to become pregnant, and weight gain control during pregnancy all serve as short-term interventions that can have long-term effects on offspring life span and health span. A 9-month period serves as a relatively short investment of time in which modifiable risks can be corrected and have exponentially larger effects on the health and even life span of offspring.

\section{Acknowledgments}

K.J.P. was supported by US National Institutes of Health (NIH) grants (from the National Institute of Environmental Health Sciences, P42ES007380, the National Institute of Diabetes and Digestive and Kidney Diseases, R01DK090460, and the National Cancer Institute, R03CA165086). L.J.R. was supported by an American Heart Association Post-Doctoral Fellowship (15POST25110002). We intended to include a brief overview of the literature in this wide-ranging field and apologize to any authors whose work was omitted.

\section{References}

1 Wadhwa PD, Buss C, Entringer S, Swanson JM: Developmental origins of health and disease: brief history of the approach and current focus on epigenetic mechanisms. Semin Reprod Med 2009;27:358-368.

-2 Kitahara CM, Flint AJ, Berrington de Gonzalez A, Bernstein L, et al: Association between class III obesity (BMI of $40-59 \mathrm{~kg} / \mathrm{m}^{2}$ ) and mortality: a pooled analysis of 20 prospective studies. PLoS Med 2014;11:e1001673.

- $3 \mathrm{Gu} \mathrm{K}$, Cowie CC, Harris MI: Mortality in adults with and without diabetes in a national cohort of the U.S. population, 1971-1993. Diabetes Care 1998:21:1138-1145.

4 Blaize AN, Pearson KJ, Newcomer SC: Impact of maternal exercise during pregnancy on offspring chronic disease susceptibility. Exerc Sport Sci Rev 2015;43:198-203.

5 Hales CN, Barker DJ: Type 2 (non-insulindependent) diabetes mellitus: the thrifty phenotype hypothesis. Diabetologia 1992;35 595-601.

-6 Barker DJ, Gluckman PD, Godfrey KM, Harding JE, Owens JA, Robinson JS: Fetal nutrition and cardiovascular disease in adult life. Lancet 1993;341:938-941.

7 Holliday R: Epigenetics: a historical overview. Epigenetics 2006;1:76-80.

8 Bunt JC, Tataranni PA, Salbe AD: Intrauterine exposure to diabetes is a determinant of hemoglobin $\mathrm{A}(1) \mathrm{c}$ and systolic blood pressure in pima Indian children. J Clin Endocrinol Metab 2005;90:3225-3229.

\9 Pettitt DJ, Baird HR, Aleck KA, Bennett PH, Knowler WC: Excessive obesity in offspring of Pima Indian women with diabetes during pregnancy. N Engl J Med 1983;308:242-245.

10 Dabelea D, Knowler WC, Pettitt DJ: Effect of diabetes in pregnancy on offspring: follow-up research in the Pima Indians. J Matern Fetal Med 2000;9:83-88.

11 Simeoni U, Barker DJ: Offspring of diabetic pregnancy: long-term outcomes. Semin Fetal Neonatal Med 2009;14:119-124.

$\checkmark 12$ Ma RC, Tutino GE, Lillycrop KA, Hanson MA, Tam WH: Maternal diabetes, gestational diabetes and the role of epigenetics in their long term effects on offspring. Prog Biophys Mol Biol 2015;118:55-68.
13 Lustig RH: Obesity before Birth: Maternal and Prenatal Influences on the Offspring. New York, Springer, 2011.

14 Olson CM, Strawderman MS, Dennison BA: Maternal weight gain during pregnancy and child weight at age 3 years. Matern Child Health J 2009;13:839-846.

15 Poston L: Maternal obesity, gestational weight gain and diet as determinants of offspring long term health. Best Pract Res Clin Endocrinol Metab 2012;26:627-639.

16 Painter RC, Roseboom TJ, Bleker OP: Prenatal exposure to the Dutch famine and disease in later life: an overview. Reprod Toxicol 2005;20:345-352.

$\checkmark 17$ Barker DJ, Hales CN, Fall CH, Osmond C, Phipps K, Clark PM: Type 2 (non-insulin-dependent) diabetes mellitus, hypertension and hyperlipidaemia (syndrome X): relation to reduced fetal growth. Diabetologia 1993;36:6267.

18 Heijmans BT, Tobi EW, Stein AD, Putter H, Blauw GJ, Susser ES, Slagboom PE, Lumey LH: Persistent epigenetic differences associated with prenatal exposure to famine in humans. Proc Natl Acad Sci USA 2008;105: 17046-17049.

19 Thomas C, Hypponen E, Power C: Prenatal exposures and glucose metabolism in adulthood: are effects mediated through birth weight and adiposity? Diabetes Care 2007;30: 918-924.

20 Painter RC, De Rooij SR, Bossuyt PM, Osmond C, Barker DJ, Bleker OP, Roseboom TJ: A possible link between prenatal exposure to famine and breast cancer: a preliminary study. Am J Hum Biol 2006; 18:853-856.

21 de Rooij SR, Wouters H, Yonker JE, Painter RC, Roseboom TJ: Prenatal undernutrition and cognitive function in late adulthood. Proc Natl Acad Sci USA 2010;107:16881-16886.

22 Kaati G, Bygren LO, Edvinsson S: Cardiovascular and diabetes mortality determined by nutrition during parents' and grandparents' slow growth period. Eur J Hum Genet 2002; 10:682-688.
23 Bygren LO, Kaati G, Edvinsson S: Longevity determined by paternal ancestors' nutrition during their slow growth period. Acta Biotheor 2001;49:53-59.

24 Pembrey M: Imprinting and transgenerational modulation of gene expression; human growth as a model. Acta Genet Med Gemellol (Roma) 1996;45:111-125.

-25 Veenendaal MV, Painter RC, de Rooij SR, Bossuyt PM, van der Post JA, Gluckman PD, Hanson MA, Roseboom TJ: Transgenerational effects of prenatal exposure to the 1944-45 Dutch famine. BJOG 2013;120:548-553.

26 Chambers TJ, Morgan MD, Heger AH, Sharpe RM, Drake AJ: High-fat diet disrupts metabolism in two generations of rats in a parent-of-origin specific manner. Sci Rep 2016;6:31857.

27 Reynolds LJ, Dickens BJ, Green BB, Marsit CJ, Pearson KJ: Using neonatal skin to study the developmental programming of aging. Exp Gerontol 2017;94:93-98.

28 Samuelsson AM, Matthews PA, Argenton M, Christie MR, McConnell JM, Jansen EH, Piersma AH, Ozanne SE, Twinn DF, Remacle C, Rowlerson A, Poston L, Taylor PD: Dietinduced obesity in female mice leads to offspring hyperphagia, adiposity, hypertension, and insulin resistance: a novel murine model of developmental programming. Hypertension 2008;51:383-392.

29 Alfaradhi MZ, Fernandez-Twinn DS, MartinGronert MS, Musial B, Fowden A, Ozanne SE: Oxidative stress and altered lipid homeostasis in the programming of offspring fatty liver by maternal obesity. Am J Physiol Regul Integr Comp Physiol 2014;307:R26-R34.

30 Taheripour P, DeFord MA, Arentson-Lantz EJ, Donkin SS, Ajuwon KM, Newcomer SC: Impact of excess gestational and post-weaning energy intake on vascular function of swine offspring. BMC Pregnancy Childbirth 2014;14:405.

31 Harman D, Eddy DE: Free radical theory of aging: beneficial effect of adding antioxidants to the maternal mouse diet on life span of offspring; possible explanation of the sex difference in longevity. Age 1979;2:109-122. 
-32 Sun L, Sadighi Akha AA, Miller RA, Harper JM: Life-span extension in mice by preweaning food restriction and by methionine restriction in middle age. J Gerontol A Biol Sci Med Sci 2009;64:711-722.

- 33 Ozanne SE, Hales CN: Lifespan: catch-up growth and obesity in male mice. Nature 2004;427:411-412.

- 34 Fagundes AT, Moura EG, Passos MC, Oliveira E, Toste FP, Bonomo IT, Trevenzoli IH, Garcia RM, Lisboa PC: Maternal low-protein diet during lactation programmes body composition and glucose homeostasis in the adult rat offspring. Br J Nutr 2007;98:922-928.

-35 Sadagurski M, Landeryou T, Blandino-Rosano M, Cady G, Elghazi L, Meister D, See L, Bartke A, Bernal-Mizrachi E, Miller RA: Long-lived crowded-litter mice exhibit lasting effects on insulin sensitivity and energy homeostasis. Am J Physiol Endocrinol Metab 2014;306:E1305-E1314.

- 36 Miller RA, Harper JM, Galecki A, Burke DT: Big mice die young: early life body weight predicts longevity in genetically heterogeneous mice. Aging Cell 2002;1:22-29.

-37 Davis K, Chamseddine D, Harper JM: Nutritional limitation in early postnatal life and its effect on aging and longevity in rodents. Exp Gerontol 2016;86:84-89.

-38 Eriksson JG, Forsen T, Tuomilehto J, Winter PD, Osmond C, Barker DJ: Catch-up growth in childhood and death from coronary heart disease: longitudinal study. BMJ 1999;318: 427-431.

- 39 Chen JH, Martin-Gronert MS, Tarry-Adkins J, Ozanne SE: Maternal protein restriction affects postnatal growth and the expression of key proteins involved in lifespan regulation in mice. PLoS One 2009;4:e4950.

40 Power C, Jefferis BJ: Fetal environment and subsequent obesity: a study of maternal smoking. Int J Epidemiol 2002;31:413-419.

-41 Rayfield S, Plugge E: Systematic review and meta-analysis of the association between maternal smoking in pregnancy and childhood overweight and obesity. J Epidemiol Community Health 2017;71:162-173.

-42 Rashid CS, Carter LG, Hennig B, Pearson KJ: Perinatal polychlorinated biphenyl 126 exposure alters offspring body composition. J Pediatr Biochem 2013;3:47-53.

43 Chen H, Iglesias MA, Caruso V, Morris MJ: Maternal cigarette smoke exposure contributes to glucose intolerance and decreased brain insulin action in mice offspring independent of maternal diet. PLoS One 2011; 6:e27260.
Curtin SC, Matthews TJ: Smoking prevalence and cessation before and during pregnancy: data from the birth certificate, 2014. Natl Vital Stat Rep 2016;65:1-14.

45 Ghosh S, Murinova L, Trnovec T, Loffredo CA, Washington K, Mitra PS, Dutta SK: Biomarkers linking PCB exposure and obesity. Curr Pharm Biotechnol 2014;15:1058-1068.

46 Plant DT, Pawlby S, Sharp D, Zunszain PA, Pariante CM: Prenatal maternal depression is associated with offspring inflammation at 25 years: a prospective longitudinal cohort study. Transl Psychiatry 2016;6:e936.

- 47 Entringer S, Wust S, Kumsta R, Layes IM, Nelson EL, Hellhammer DH, Wadhwa PD: Prenatal psychosocial stress exposure is associated with insulin resistance in young adults. Am J Obstet Gynecol 2008;199:498 e491e497.

48 King KE, Kane JB, Scarbrough P, Hoyo C, Murphy SK: Neighborhood and family environment of expectant mothers may influence prenatal programming of adult cancer risk: discussion and an illustrative DNA methylation example. Biodemography Soc Biol 2016; 62:87-104.

49 Brunton PJ: Effects of maternal exposure to social stress during pregnancy: consequences for mother and offspring. Reproduction 2013; 146:R175-R189.

50 Gavrilov LA, Gavrilova NS: Season of birth and exceptional longevity: comparative study of American centenarians, their siblings, and spouses. J Aging Res 2011;2011:104616.

51 Chen Z, Toth T, Godfrey-Bailey L, Mercedat N, Schiff I, Hauser R: Seasonal variation and age-related changes in human semen parameters. J Androl 2003;24:226-231.

52 Gavrilov LA, Gavrilova NS: Early-life programming of aging and longevity: the idea of high initial damage load (the HIDL hypothesis). Ann NY Acad Sci 2004;1019:496-501.

53 Gavrilov LA, Gavrilova NS: New developments in the biodemography of aging and longevity. Gerontology 2015;61:364-371.

54 Gavrilova NS, Gavrilov LA: Parental age at conception and offspring longevity. Rev Clin Gerontol 1997;7:5-12.

55 Mills M, Rindfuss RR, McDonald P, te Velde E, Reproduction E, Society Task F: Why do people postpone parenthood? Reasons and social policy incentives. Hum Reprod Update 2011;17:848-860.

56 Carnes BA, Riesch R, Schlupp I: The delayed impact of parental age on offspring mortality in mice. J Gerontol A Biol Sci Med Sci 2012; 67:351-357.
57 Martens DS, Plusquin M, Gyselaers W, De Vivo I, Nawrot TS: Maternal pre-pregnancy body mass index and newborn telomere length. BMC Med 2016;14:148.

58 Njajou OT, Cawthon RM, Damcott CM, Wu SH, Ott S, Garant MJ, Blackburn EH, Mitchell BD, Shuldiner AR, Hsueh WC: Telomere length is paternally inherited and is associated with parental lifespan. Proc Natl Acad Sci USA 2007;104:12135-12139.

59 Carter LG, Lewis KN, Wilkerson DC, Tobia CM, Ngo Tenlep SY, Shridas P, Garcia-Cazarin ML, Wolff G, Andrade FH, Charnigo RJ, Esser KA, Egan JM, de Cabo R, Pearson KJ: Perinatal exercise improves glucose homeostasis in adult offspring. Am J Physiol Endocrinol Metab 2012;303:E1061-E1068.

60 Carter LG, Qi NR, De Cabo R, Pearson KJ: Maternal exercise improves insulin sensitivity in mature rat offspring. Med Sci Sports Exerc 2013;45:832-840.

61 Sheldon RD, Nicole Blaize A, Fletcher JA, Pearson KJ, Donkin SS, Newcomer SC, Rector RS: Gestational exercise protects adult male offspring from high-fat diet-induced hepatic steatosis. J Hepatol 2016;64:171-178.

-62 Carter LG, Ngo Tenlep SY, Woollett LA, Pearson KJ: Exercise improves glucose disposal and insulin signaling in pregnant mice fed a high fat diet. J Diabetes Metab 2015;6: 634.

63 Newcomer SC, Taheripour P, Bahls M, Sheldon RD, Foust KB, Bidwell CA, Cabot R: Impact of porcine maternal aerobic exercise training during pregnancy on endothelial cell function of offspring at birth. J Dev Orig Health Dis 2012;3:4-9.

64 May LE, Glaros A, Yeh HW, Clapp JF 3rd, Gustafson KM: Aerobic exercise during pregnancy influences fetal cardiac autonomic control of heart rate and heart rate variability. Early Hum Dev 2010;86:213-217.

65 Camarillo IG, Clah L, Zheng W, Zhou X, Larrick B, Blaize N, Breslin E, Patel N, Johnson D, Teegarden D, Donkin SS, Gavin TP, Newcomer S: Maternal exercise during pregnancy reduces risk of mammary tumorigenesis in rat offspring. Eur J Cancer Prev 2014;23:502505 .

66 Herring A, Donath A, Yarmolenko M, Uslar E, Conzen C, Kanakis D, Bosma C, Worm K, Paulus W, Keyvani K: Exercise during pregnancy mitigates Alzheimer-like pathology in mouse offspring. FASEB J 2012;26:117-128.
Perinatal Influences on Disease Development
Gerontology 2018;64:237-245 DOI: $10.1159 / 000485506$ 